\title{
EFEITO DO EXTRATO AQUOSO BRUTO DE Polygonum acre H.B.K. SOBRE A MUSCULATURA LISA E CORRELAÇĀO COM SUA COMPOSIÇĀO QUIMIICA
}

Augusto Vilson Bortoluzzi; Marilia Appel de M. Bortoluzzi e Ana Maria Chagas

Departamento de Farmācia Industrial e de Fisiologia - Núcleo de Pes quisas de Produtos Naturais. Centro de Ciências Naturais e Exatas. UFSM. Santa Maria, RS.

Janice Sityā e Licērio Vicente Padoim

Curso de Medicina. Centro de Ciências da Saūde. UFSM. Santa Maria-RS.

RESUMO

Polygonum acre H.B.K., planta perene de lugares úmidos e pantanosos, muito abundante no Brasil, ē utilizada em medicina popu lar como anti-hemorrāgica e expectorante. Os autores objetivaram es tudar o extrato aquoso bruto das folhas do vegetal a $20 \%$ sobre a musculatura lisa, utilizando ūtero de ratas em estro (estilbestrol) e correlacionar este efeito com a composição química do vegetal.

Para esclarecer o efeito sobre a contrabilidade do múscu 10 liso, utilizou-se 38 ūteros isolados de ratas, em banho-maria de Magnus, empregando-se transductor de força acoplado ao fisiōgrafo (marca Harvard, U.S.A.). As experiēncias foram realizadas em prepa rações de ütero conforme tēcnica descrita acima. As concentrações tes tadas atravēs de adição ao banho, selecionadas em experiēncia pilo to, foram de 0,$5 ; 1,0 ; 2,0 \mathrm{ml}(0,2 \mathrm{~g} / \mathrm{ml})$ de extrato aquoso bruto de Polygonum acre H.B.K. e de adrenalina utilizou-se uma dose de $3 \times 10^{-6}$

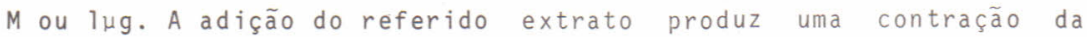
musculatura uterina, mas esta não é significativa comparando-se com a ação de adrenalina. Em relação à frequência, o nūmero de contrações tambēm não foi significativo. Sabemos que o vegetal possui flavonó $\underline{i}$ des que poderiam ser responsāveis pelo efeito constritor sobre a musculatura lisa, atravēs de uma ação direta ou indireta. Nas con dições experimentais utilizadas, concluimos que, em relação a esta planta, a ação não deve ser direta.

SUMMARY

BORTOLUZZI, A.V.; BORTOLUZZI; M.A.M.; CHAGAS, A.M.; SITYA, J. and PADOIN, L.V., 1989. Effect of crude aqueous extract of Polygonum acre H.B.K. on the smooth muscle and their correlation with the chemical composition. Ciência e Natu ra 11: $93-99,1989$

Polygonum acre H.B.K. perennial native plant from humid and swampy places, very abundant in Brazil, is used in popular medicine as antihemorrhagic and as expectorant. The authors objective were to 
study the $20 \%$ crude aqueous extract of the vegetable leaves on smooth muscle using rats uterus in oestrus (stilboestrol) and to verify the correlaction of this effect with the chemical composition. For this purpose, we used rats uterus preparation in Magnus bath with De Jalon's solution aerated. Those concentration were choosen from pilot experiences and were 0,$5 ; 1,0$ and $2,0 \mathrm{ml}(0,2 \mathrm{~g} / \mathrm{ml})$ of the crude aqueous extract of the $P$. acre H.B.K. and the adrenaline doses were $1,0 \mu \mathrm{g}$. The results showed that the contraction of the smoothmuscle and the alteration of the frequence produced by the extract were not significant, this plant flavonoids, which could be responsable by the constriction effect on the smooth muscle, perhaps by one direct or indirect action. This suggests that the effect of the crude extract are possibly due to the indirect action.

INTRODUÇAO

Polygonum acre H.B.K., pertencente a famīlia Polygonaceae, conhecida popularmente como "erva-de-bicho", entre outros como "per sicaria do Brasil", é uma planta perene que se desenvolve em lugares úmidos e pantanosos muito abundante no Brasil (14).

o gēnero Polygonum abrange vārias espēcies dispersas em regiões tropicais e subtropicais do globo (10). A espécie objeto des te estudo é sinōnimo de Polygonum antihaemorrhoidale var. aquatize Mart., Polygonum hydropiper Michaux., Polygonum hidropiperoides Pursh., Polygonum alatum Ham. e Polygonum punctatum Elliot.

0 interesse pelo estudo do vegetal fundamentou-se no seu uso em medicina popular para vārios fins, principalmente como antihemorroidal, anti-hemorrágico, antidiarréico e expectorante (10).

o comércio farmacêutico usa, com frequência, o vegetal em formulações devido as suas propriedades anti-hemorrágicas, bem como em associações com drogas antiespasmódicas e sedativas como a sela dona, nas crises hemorroidārias.

Vārios grupos quỉmicós jā foram detectados no vegetal, en tre eles flavonóides (4, 15), agliconas tipo flavonol, como querce tina, campferol, isoramnetina e ramnazina (18), isocumarinas, como a poligonolida, com atividade anti-inflamatória, isolada das raizes de $P$. hydropiper (8).

A partir do extrato metanōlico das raizes da mesma espécie foi isolado um cumaril-glicosỉdeo chamado hidropiperosídeo, além de antraquinonas, ācido elágicó 3,3'-di-0-metil-ēter, ācido gālico, 2 glicosīdeos da quercetina e uma gama-lactona aromática com ativida de antifērtil (7).

Nas folhas encontram-se vārios sesquiterpenos e norsesqui terpenos, entre eles, um sesquiterpeno tipo drimano, dialdeido, com potente atividade citotóxica, antibiótica e moluscicida, owarburganal, 
e o poligodial com atividade anticomplemento $(10,5 \mathrm{~g} / \mathrm{ml})$, além de um norsesquiterpeno monoaldeídico, o poligonal (5). Outro sesquiter peno presente no vegetal é o ácido poligonico, que tambēm possui at vidade anticomplemento, porēm, esta è de menor intensidade (6).

0 vegetal possue vārios ácidos fenólicos, entre eles, os ācidos cis/trans p-cumārico, cis/trans ferūlico, cis/trans sināpi co, vanîlico, p-hidroxibenzóico, cis/trans cafeico, clorogênico e salicîlico (13).

SHALAMOVA e colaboradores (12) estudaram 3 espécies de Po Zygonum e detectaram em $P$. hydropiper alto teor de taninos, alēm de vitamica C, ácidos förmico, acético, tartārico, valérico, glicurôni co e galacturônico e verificaram que os nỉveis de potássio, sōdio, cálcio e magnésio são de três a quatro vezes maiores nas cinzas dos polissacarídeos isolados do vegetal, do que nas cinzas do mesmo.

0 conteúdo polissacarídico è máximo durante a floração, sen do que seus principais componentes são o ácido galacturônico e a ramnose, enquanto os principais monossacarídeos são a galactose, gli cose, manose, arabinose e xilose. Devido a sua composição em monos sacarỉdeos, o caráter de sua hidrōlise àcida e seu alto nívelem ácí do galacturônico, o vegetal pode ser classificado como pectinas (17).

Em relação a estabilidade do extrato fluido de $P$. hydrop $\underline{i}$ per, PLUTA e colaboradores (11) verificaram que a luz não apresenta efeito significante sobre a turvação do extrato, sendo que frascos de vidro àmbar protegem suficientemente as preparações.

Em virtude deste vegetal ser utilizado em medicina popular como anti-hemorrágico, e atravēs dos estudos jā realizados à respei to de sua composição quỉmica, ter sido detectado a presença de fla vonóides, os autores objetivaram avaliar a atividade do extrato aquo so bruto sobre a musculatura 1 isa, cuja ação poderā explicar o meca nismo deste efeito medicinal.

\section{MATERIAL E METODOS}

Material vegetal: O vegetal foi coletado no Distrito Boza no, Ijuî, RS, em agosto de 1988, identificado pelo Depto. de Biolo gia da UFSM, onde encontra-se exsicata do mesmo. As fol has do vege tal foram separadas, dessecadas à $50^{\circ} \mathrm{C}$ e reduzidas à pó em macromoinho tipo Wyllie.

Preparação dos extratos: Foi preparado extrato aquoso das fol has do vegetal, a $20 \%$, pelo método de decocção.

Procedimento: As experiências foram realizadas empregan do-se ratas Wistar, virgens, em estro, pesando aproximadamente $200 \mathrm{~g}$, e a técnica adotada para estudo sobre a musculatura lisa, foi a de útero isolado, em banho-maria de Magnus, com De JALON aerado (2) e, fundamentalmente, é desenvolvida conforme os seguintes pontos: os animais receberam, via intraperitonial, $0,1 \mathrm{mg} / \mathrm{kg}$ de estilbestrol e 
apōs, foram sacrificados com uma pancada na região cervical, efetuan do-se, imediatamente, laparatomia com a retirada do ūtero (histerec tomia). Os ūteros, após a retirada da serosa excedente, foram colo cados no banho de Magnus. As contrações foram registradas atravēs de um transdutor de força acoplado a um eletrofisiōgrafo (marca Harvard, USA).

Para investigar a sensibilidade da preparação, utilizou-se 0,1 e/ou $0,2 \mathrm{ml}$ de acetilcolina $10^{-6} \mathrm{M}$ no banho de Magnus e, após 0 resultado, a preparação foi lavada 3 vezes.

A seguir foi colocado 0,$5 ; 1,0 ; 2,0 \mathrm{ml}$ de extrato aquoso bruto das folhas do vegetal à $20 \%, 10 \mathrm{go}, 0,2 \mathrm{~g} / \mathrm{ml}$, e $3 \times 10^{-6}$ de adre nalina.

Os registros foram avaliados pelo teste $t$ de student para um nỉvel de significância de 0,5 (3).

\section{RESULTADOS}

0 extrato aquoso do vegetal produziu contrações maiores que as contrações normais, no entanto, menores que as produzidas pelos mediadores quimicos empregados, acetilcolina e adrenalina. (Fig. 1).

A frequência das contrações também não foi alterada signi ficativamente conforme se observa na Fig. 2.

\section{I SCUSSAOO}

Analisando-se os resultados referentes ao extrato aquoso, observou-se que não foram dose-dependente, isto $\bar{e}$, não houve contra ções e nem alteração da frequência significativas, mesmo com o aumen to das doses. Estas contrações são significativamente menores que as produzidas pelos mediadores químicos empregados.

Em relação à composição quỉmica do vegetal, vārios grupos de constituintes estão presentes, entre eles, flavonóides, os quais possuem ação sobre a rede capilar, diminuindo a permeabilidade e a fragilidade dos vasos.

o mecanismo de ação dos flavonōides é discutido, podendo ser por uma ação constritora indireta, segundo CLARK \& GEISSMAN (1) ou WILSON e DE EDS.(16), uma vez que os flavonóides formam sistemas de oxidação e são agentes queladores e, alguns, "in vitro", jā foi demonstrado, que inibem a oxidação da adrenalina, aumentando a con centração desta na circulação ou, mais provavelmente, que os flavo nōides exerçam uma ação vaso constritora direta sobre os capilares, diminuindo a permeabilidade, o que pode explicar também, os efeitos sobre a fragilidade capilar deste grupo de compostos (9).

o extrato não apresentou atividade acentuada sobre a mus culatura lisa, como era de se esperar, por isto, pode-se descartar, nas condições estudadas, uma ação constritora direta referente aos flavonóides. Nossos resultados nos levam a acreditar na possibilidade 
de uma ação indireta.

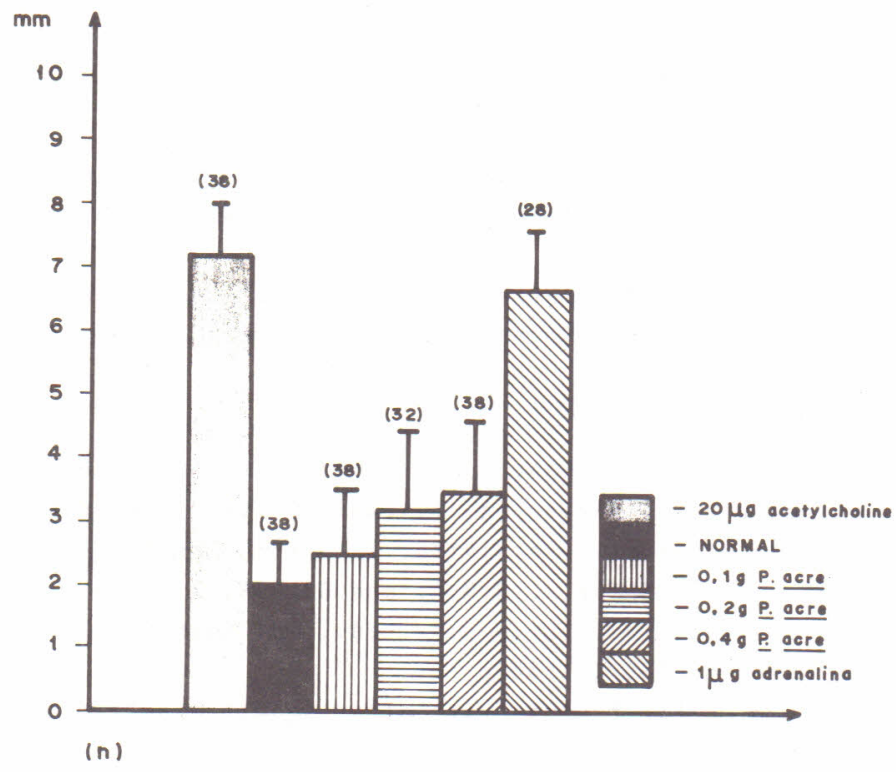

Figura 1 - Efeito do extrato aquoso bruto de Polygonum acre H.B.K. acetilcolina e adrenalina, sobre a contração uterina, ex pressa em milizmetros.

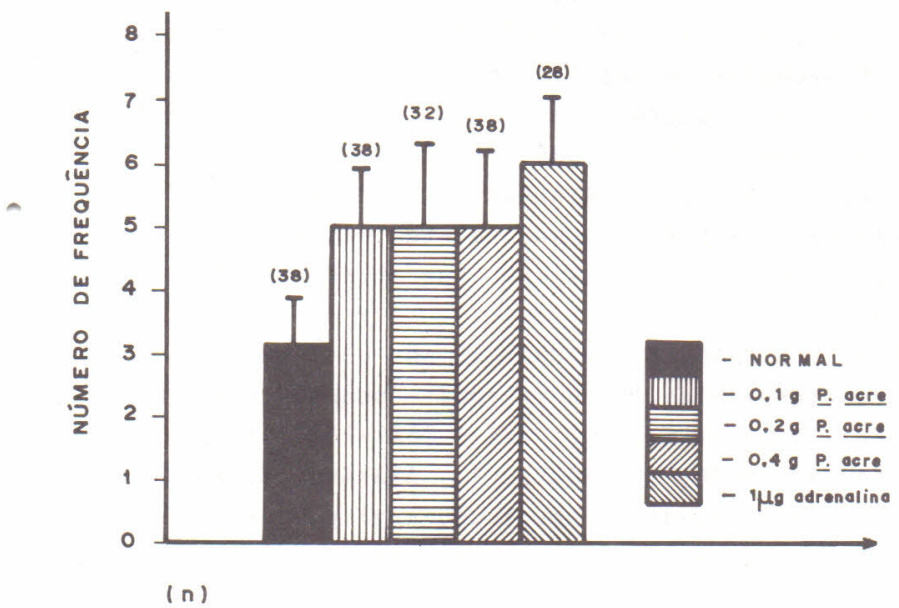

Figura 2 - Efeito do extrato aquoso bruto de Polygonum acre H.B.K, e adrenalina, sobre a frequência da contração uterina.

Por outro lado, deve-se ter em mente, que a presente inves tigação foi desenvolvida empregando-se extrato aquoso bruto e, tal vez, estas condições possam mascarar os resultados. 
Este aspecto, pretende-se avaliar posteriormente, utilizan do-se extratos purificados contendo somente a fração flavonoỉdica de Polygonum acre H.B.K.

CONCLUSÕES

0 extrato aquoso bruto das fol has de Polygonum acre H.B.K. não apresenta atividade significativa sobre a musculatura 1 isa, no modelo estudado.

A presença de flavonóides no vegetal, que caracteriza a ação constritora, provavelmente ocorra por uma ação indireta.

\section{REFERENCIAS BIBLIOGRAFICAS}

1. CLARK, W.G. and GEISSMAN, T.A. - Potentiation of effects of epinephrine by flavonoid ("vitamin P-like") compounds. Rela tion of struture to activity. J. Pharmac. Exp. Ther, 95: 363-381, 1949, in: GOODMANN, L.S.M.A. and GILMAN, A. - As bases farma cológicas da terapêutica. 3a. ed., Ed. Guanabara Koogan, Rio de Janeiro, 1967, 1530 p.

2. DE JALON, B. - Experiments with other smooth muscle preparations, in: PERRY, W.L.M. - Pharmacological Experiments on Isolated Preparations. 2a. ed., E. \& S. Livingstone, London, 1970,88$111 \mathrm{p}$.

3. DIXON, W.J. and MASSEY Jr., F.S.J. - Introduction to Statistical Analyses. MacGraw Hi11, New York, 1969, 122p.

4. FETKHULLINA, G.A. and BULENKOV, T. I. - Quantitative determination of flavonoids in the liquid extract of Polygonum hydropiper and Hypericum tincture. Farmatsiya, 33(5):38-40, 1984, in: Biol. Abst. $79(6): 53064,1984$.

5. FUKUYAMA, Y.; SATO, T.; ASAKAWA, Y. and TAKEMOTO, T. - A potent cytotoxic warburganal and related drimane-type sesquiterpenoids from Polygonum hydropiper. Phytochemistry. 21(12):2895-2898, 1982 .

6. FUKUYAMA, Y.; SATO, T.; MIURA, I. and ASAKAWA, Y. - Drimane-type sesquiterpenoids and norsesquiterpenoids from Polygonum hydro piper. Phytochemistry, 24(7): 1521-1524, 1985.

7. FUKUYAMA, Y.; SATO, T.; MIURA, I.; ASAKAWA, Y. and TAKEMOTO, T. - Hydropiperoside, a novel coumaryl glycoside from the root of Polygonum hydropiper. - Phytochemistry, 22 (2):549- 552, 1983.

8. FURUTA, T.; FUKUYAMA, Y. and ASAKAWA, Y. - Polygonolide, an iso coumarin from Polygonum hydropiper possessing anti-inflammatory activity. Phytochemistry, 25(2):517-520, 1986.

9. GOODMANN, L.S.M.A. and GILMAN, A. - As bases farmacológicas da terapêutica. 3a. ed., Ed. Guanabara Koogan, Rio de Janeiro, 
$1967,1529-1531 \mathrm{p}$.

10. HOEHNE, F.C. - Plantas e substâncias vegetais tóxicas e medici nais. 2a. ed., Novos Horizontes Ltda., São Pau1o, 1978, 108$109 \mathrm{p}$.

11. PLUTA, J.; OLSZEWSKI, A. and ZABA, A. - Influence of physicoche mical and technological agents on precipitate formation in the fluid extract of Polygonum hydropiper. Herba Pol., 31 $(3 / 4)$ : 201-208, 1985 (1987), in: Biol. Abst., 85(11),114354, 1985 (1987).

12. Shalamova, G.g.; yakovlev, A. I. and Churilov, G.i. - Chemical composition of epigeal parts of 3 Polygonum spp. - Rastit Resur., 19(4): 526-529, 1983, in: Biol. Abst., 78(9): 70800, 1983.

13. SWIATEK, L. and DOMBROWiCZ, E. - Phenolic acids in medicinal plant drugs of Polygonum spp. - Farm. Pol., 43(7-8): 420-433, 1987, Chem. Abst. 108, 156523g.

14. THOMSON, W.A.R. - Guia practico ilustrado de las plantas medici nales. Ed. Blume, Barcelona, 1981, 89 e 182p.

15. VYSOCHINA, G.I.; KUL'PINA, T.G. and BEREZOVSKAYA, T.P. - Flavo noid content in some Polygonum spp. of the section Persicari (Mi11.) DC in the flora of Siberia. Rastit. Resur., 23 (2): 229-234, 1987, in: Biol. Abst., 85(1):7746, 1987.

16. WILSON, R.H. and DE EDS, F. - The in vitro protection of epine phrine by flavonoids - J. Pharmac. Exp. Ther, 95,399-406, 1949, in: GOODMANN, L.S.M.A. and GILMAN, A. - As bases farmacológ $\underline{i}$ cas da terapêutica. 3a. ed., Ed. Guanabara Koogan, Rio de Ja neiro, 1967, 1530p.

17. YAKOVLEV, A.I. and CHURILOV, G.I. - Characteristics and dynamics - of polysaccharide content in Polygonum hydropiper epigeal parts. Rastit Resur., 19(1):68-71, 1983, in: Biol. Abst. ?? (9): $70750,1983$.

18. YUSIF, K.S. and BLINOVA, K.F. - Flavonol aglycons of Polygonum hydropiper. Khim. Prir. Soedin., (5), 658-659, 1984, in: Chem. Abstr., 102, 42886y, 1985.

Recebido em outubro 1989, aceito em dezembro, 1989. 
